subjects which the editor attempts to cover, and the fact that so few reviews actually appear in each number. mean that the specialist reader will be lucky to find even one article of specific interest in a year's issues. Sccondly, the way in which the issues are produced suggests that the interval between the submission of a manuscript and its eventual appearance in print may be quite substantial, perhaps more than a year. For some subjects this may not matter too much - I was fascinated to read that carly allusions to fibrinolysis can be found in the work of Hippocrates but in other, fast-moving areas such delays can be frustrating. These reservations notwithstanding, the scientific standard is undoubtedly high and all medical libraries will need to subscribe.

The scope of Hematological Oncology, defined in the editorial in the first issue, is also wide, not to say formidable "pathology, radiodiagnosis, radiation

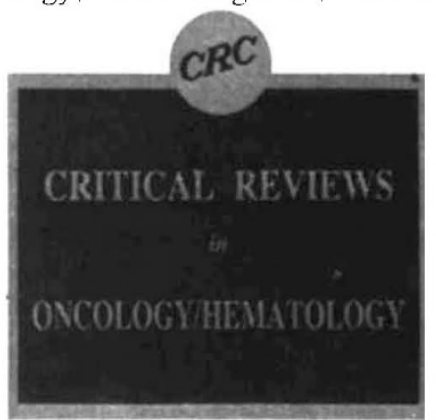

therapy, medical oncology, immunology, virology, epidemiology, tissue culture, in vitro drug testing. cytogenetics, cell kinetics, receptor assays. HLA studies and other related arcas", to mention only some of the topics. This must mean that the journal will consider virtually any contribution in the field of oncology, though in practice the contents reflect the editors particular interests (the immunopathology, clinical features and treatment of lymphoproliferative disorders). Papers on cell lines, cytogenetics and oncogenic viruses are also well represented.

The bulk of each issue consists of original research papers of somewhat variable quality, but the editors have solicited and published a number of valuable review articles--on simian AIDS, the Kiel classification of non-Hodgkin`s lymphoma and purging bone marrow before autografting, for example. Also included are short communications. book reviews and a forum in which individual topics are addressed by chosen authors: letters from readers are invited. It appears that the time from acceptance to publication is agreeably short.

Overall. this journal looks like being a useful addition to the oncological literature. But perhaps its precise content needs to be somewhat more narrowly defined for the future.

Joln M. Goldman is in the Medicat Researd Council's Leakacmia Unit at the Roval Postgraduate Medical School. Hammersmith Hospital. Dacane Road. London WI2 ()HS. UK.

\section{Cosmopolitan agents in context}

\section{B.L. Ginsborg}

Biogenic Amines: The International Interdisciplinary Journal Reporting Basic and Clinical Research on Biogenic Amines and Aminoacid Transmitters. Chief editors H. Parvez and T. Nagatsu. $V N U$ Science Press. 6/yr. Surface DM 378, air DM 390.

Biogenic Amines is published in Utrecht its price is set in deutschmark; its editorsin-chief are Parvez from Paris and Nagatsu from Nagoya; and its editorial board of some 50 members is drawn from eleven different countries, and includes Axelrod from Bethesda, Sandler from London, Lapin from Leningrad and Seeman from Toronto. In a sample of four issues, amounting to about 320 pages, thirteen articles are from Japan, five from the GDR and the remaining fifteen from: eleven other countries.

Among the titles of papers in the sample are "Oxidation of acetylpolyamines by amine oxidases from soybean and oat seedlings", "A sensitive assay for Bphenylethylamine in biological samples by gas chromatography/chemical ionization mass spectrometry", "Tyramine-induced suppression of central noradrenergic neurons following monoamine oxidase inhibition" and "Elevated levels of polyamines and radioimmunoassayable 6hydroxymethylpterin-like compound in urine from cancer patients". The dominant subject of investigation is the rat (seventeen articles) but also studied are man (five articles), the goat, chicken, dog, goldfish, rabbit and bovine adrenal medulla. From all this it is evident that the publisher's description of the journal as "international and interdisciplinary reporting basic and clinical research" would not contravene any tradedescription act.

The papers are in English, and have the standard format of introduction. methods, results, discussion and refer-

\section{BIOGENIC AMINES}

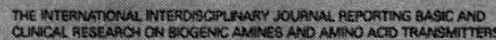

ences. There are no page charges. Clearly, Biogenic Amines could provide an alternative home for papers which might otherwise have appeared, at least in respect of their subject matter, in established journals of biochemistry, pharmacology, analytical methods or neuroscience. But my guess is that authors will generally continue to send their more exciting work to a journal of the appropriate discipline.

It is true that there are successful journals concerned exclusively with particular classes of agents of biological interest. such as cyclic nucleotides, neuropeptides and prostaglandins to mention but three. However these are substances in their adolescence which attract enthusiastic. single-minded followers determined to assign to them as many biological functions as possible. By contrast. amines already occupy positions of undisputed importance in the biological scheme of things. They do not seem to me to require positive discrimination in the form of an exclusive publication.

B. L. Ginsborg is a Professor in the Department of Pharmacology, University of Edinburgh. I George Square, Edinburgh, EH8 9JZ, UK.

\section{Periodical changes}

\section{A. J. Leigh Brown}

Molecular Biology and Evolution. Editorin-chief Waiter $M$. Fitch. University of Chicago Press. 6/yr. North America \$60 (institutional), $\$ 30$ (individual); elsewhere $\$ 64$ (institutional), $\$ 34$ (individual).

IN RECENT years, the molecular approach to evolutionary biology has proved very fruitful. Few would dispute the interest engendered by the mass of data coming from this source, although it may be some time before our understanding of the process of adaptive evolution is greatly advanced by them. In these circumstances it is hardly surprising that a new journal should appear, although the impetus for the appearance of Molecular Biology and Evolution came from scientists, rather than a publishing company. Perhaps in consequence, the subscription price is very reasonable, but it remains to be seen whether the journal will fulfil all the hopes of its editors.

One of the intentions of the journal is to bring together articles covering the broad field of molecular aspects of evolution. Certainly, the contributions published to date might previously have appeared in a very wide range of journals so this aim has been fulfilled. They present evolutionary studies which utilize nucleic acid sequences, restriction maps, immunological distances and enzyme polymorphism, and both prokaryotic and eukaryotic systems are well represented. There are regular theoretical contributions also; these are closely related to experimental work and fit in well with the overall theme. Papers of all lengths have been published, and a few reviews, book reviews and an occasional letter to the editor have also appeared. The quality of many of the articles has been very high.

Each number of Molecular Biology and Evolution contains five or six papers. Surprisingly, the journal has not expanded since its first issues and perhaps it has not yet caught the attention of molecular biologists en masse. Some features may be 